medication. Increasing visit frequency to primary care $(\mathrm{HR}=1.85 ; 95 \% \mathrm{CI}$, $1.58-2.18)$ and endocrinology $(\mathrm{HR}=2.08 ; 95 \% \mathrm{CI}, 1.45-2.97)$ were associated with decreasing delays, as were increasing income levels ( $\mathrm{HR}=1.04 ; 95 \% \mathrm{CI}$, 1.00-1.07). Conclusions: Patients with diabetes facing inertia are at risk of further delays in appropriate management. Our findings suggest the presence of patient, physician and system barriers to appropriate care. Increased contact with the health care system may mitigate risk.

\section{Abstract C-C1-03}

Moving the Big N

Ellen Vanderboom, MSN, RN, Marshfield Clinic; Theodore Praxel, MD, MMM, FACP, Marshfield Clinic; Marilyn Follen, RN, MSN, Marshfield Clinic; Daniel Erickson, MD, Marshfield Clinic; Thomas Gabert, MD, MPH, Marshfield Clinic; Kori Krueger, MD, Marshfield Clinic; Eric Penniman, DO, Marshfield Clinic; Mary Beth Dickinson, RN, BSN, Marshfield Clinic; Trina Ford, MSN, NP, RD, Marshfield Clinic

Background: Nationally the trend for hospitalization of diabetics has been slow to show improvement. In 2003, the rate of hospital discharge for diabetes as any-listed diagnosis (360.7 per 1000 people with diabetes) was only slightly lower than the rate in 1980 (398.7 per 1000 people with diabetes). Decreasing hospitalizations and improving health outcomes for 17,678 patients has been a system wide initiative in Marshfield Clinic since 2005. Methods: Numerous interventions have occurred since 2005 to improve health outcomes and decrease costs for the diabetes population. Evidenced-based guidelines were deployed and CME activities were created for providers. Clinical information support tools and applications deployed included wireless tablets for primary care providers and staff, an electronic point of care reminder tool, medication management and electronic planned care applications were also deployed. The diabetic foot exam process was standardized and medical assistants were trained to perform foot exams. Standing orders for staff to give influenza and pneumococcal vaccinations and schedule necessary lab tests or office visits were developed. Feedback on diabetes quality metrics at the organization, division, department and individual primary care provider level was provided every quarter on a rolling 12-month basis. Results: The all-cause hospitalization rate for persons with a diagnosis of diabetes has decreased from 360 per 1000 in 2005 to 317 per 1000 in 2007. This decrease in hospitalization lead to improved economic outcomes and quality of life by avoided hospitalizations. Conservative estimates of the cost savings range from $\$ 5,202,000$ to $\$ 14,137,200$. Conclusions: Significant improvement in this population has been demonstrated system wide by a decrease in all-cause hospitalization rates and composite diabetes process and outcome measures. Use of an electronic health record is essential but not sufficient to affect change. Ongoing measurement of practice performance has assisted in the change. Physician champions have been crucial for spread. Improvements are significant, however, opportunities remain.

\section{Abstract C-C1-04 \\ The Effectiveness of Diabetes Care Management in a Large, Integrated Delivery System: a TRIAD Study}

Julie A. Schmittdiel, PhD, Kaiser Permanente Northern California Division of Research; Connie Uratsu, BA, Kaiser Permanente Northern California Division of Research; Bruce Fireman, MA, Kaiser Permanente Northern California Division of Research; Joe V. Selby, MD MPH, Kaiser Permanente Northern California Division of Research

Background: Numerous studies have examined the effect of care management (CM) programs on diabetes outcomes such as A1c control. Most evaluations have been small randomized controlled trials; few studies examine how CM translates to real-world settings in terms of success at reaching target populations, or effectiveness for improving outcomes in large populations. The aims of this project are to assess the appropriateness of entry into a diabetes (DM) nurse-delivered CM program in Kaiser Permanente Northern California (KPNC) compared with program guidelines, to determine the proportion who meet program entry criteria, and to compare the trajectory of intermediate outcomes in patients who receive $\mathrm{CM}$ to that of similar patients who don't receive CM. Methods: KPNC automated databases were used to identify all DM patients from 2000-2005 and to determine whether patients enrolled in CM. We examined whether CM enrollees and non-enrollees met the eligibility criteria for the CM program. Using propensity score matching, we matched $\mathrm{CM}$ patients to similar patients who were eligible but did not enter $\mathrm{CM}$. We compared trajectories of A1c, LDL-c, and blood pressure levels of $\mathrm{CM}$ patients to those of their matched controls, adjusting for baseline risk factor levels. Results: Between 2000 and 2005 less than 10\% of all KPNC diabetes patients entered $\mathrm{CM}$, representing approximately $25 \%$ of all eligible patients. Up to $22 \%$ of those in CM each year did not meet program entry criteria. CM patients were similar to the overall DM population in age, gender, blood pressure, and LDL-c levels; they had higher A1c and microalbumin levels. There were small but statistically significant differences in A1c, LDLc, and blood pressure levels between CM patients and their matched controls 6,12 , and 15 months after program exit. There were significant improvements in CVD risk factor levels in all KPNC DM patients over time, regardless of whether or not they received CM. Conclusions: While eligibility guidelines for diabetes nurse care management were not strictly adhered to, care management did have a small but significant impact on intermediate outcomes. However, these improvements were small compared to the risk factor improvements over time in the overall DM population.

\section{Abstract C-C1-05 \\ Who Does Not Complete a First-Fill for a Diabetes-Related Prescription?}

Nirav R. Shah, MD, MPH, Geisinger Center for Health Research; Annemarie G. Hirsch, MPH, Geisinger Center for Health Research; G. Craig Wood, MS, Geisinger Center for Health Research; Amanda F. Wingate, BS, Geisinger Center for Health Research; Ilene G. Ladd, MS, Geisinger Center for Health Research; Walter F. Stewart, PhD, MPH, Geisinger Center for Health Research

Background: Research on medication adherence is primarily conducted using insurance or pharmacy claims data, focusing on patients who have already obtained their first prescription for a defined condition. Claims data alone cannot be used to assess first-fill rates. Methods: We conducted a retrospective cohort study that examined first-fill rates for patients prescribed medications to treat diabetes by linking prescribing information from electronic health records (EHR) to pharmacy claims data of one insurer. EHR data were obtained from the Geisinger Clinic, a large group practice serving a 31-county area of central and northeastern Pennsylvania. The Clinic includes 41 community practice sites with primary and multi-specialty care, all of which have used an EHR since 2001. Claims data were obtained from Geisinger Health Plan, a payer that accounts for 30\% of Geisinger Clinic patient volume. Adherence was defined as a claim generated for the first-fill prescription within 30 days of the index date. Results: Of 1132 patients written a new, first-time prescription for any diabetes medication, $962(85 \%)$ generated a corresponding claim within 30 days. Therapeutic class, refills, co-pay, baseline A1c values, and change in Alc values were associated with first-fill rates. Gender, race, age, comorbidity score, number of office visits, and number of medications prescribed had no association $(P<0.05)$. Conclusions: Adherence relates directly to higher A1c levels and is associated with therapeutic drug class, higher number of refills, and lower co-pay. As expected, patients with higher adherence exhibited greater change in A1c levels. Patients with relatively lower baseline A1c levels, while at lower risk for diabetes complications, are at higher risk of not adhering to diabetes medications.

\section{Abstract C-C1-06 \\ Identifying Depression Among Diabetes Patients Using Natural Language Processing of Office Notes}

Lucy R. Fischer, PhD, HealthPartners Research Foundation; William A. Rush, PhD, HealthPartners Research Foundation; John C. Kluznik, PhD, HealthPartners Research Foundation; Patrick J. O'Connor, MD MPH, HealthPartners Research Foundation; Ann M. Hanson, BS, HealthPartners Research Foundation

Purpose: To compare two methods for identifying depression among health plan enrollees with diabetes - administrative data base codes versus natural language processing (NLP) of office note verbal narratives. Background: In research studies in health care systems, depression cases can be identified through coded data in the health plan administrative data system, including diagnosis (ICD-9) codes and pharmacy codes. However, depression is often 